\title{
Photodétecteurs ultra-rapides pour la métrologie d'impulsions UV-X
}

\author{
F. Foulon, P. Bergonzo, B. Guizard, A. Brambilla, D. Riedel*, \\ L. Museur* et M.C. Castex*
}

\author{
LETI, CEA-Technologies Avancées/DEIN/SPE, CEA Saclay, 91191 Gif-sur-Yvette, France \\ * Laboratoire de Physique des Lasers, Université Paris-Nord, 93430 Villetaneuse, France
}

\begin{abstract}
Résumé : Des photodétecteurs sont développés au LETIDEIN du CEA Saclay pour la métrologie d'impulsions de rayonnements VIS, UV, X et gamma, couvrant un large domaine d'énergie compris entre $1 \mathrm{eV}$ et plusieurs dizaines de MeV. Récemment, des détecteurs "solar blind" en diamant ont été mis au point pour la caractérisation d'impulsions VUV à $125 \mathrm{~nm}$ $\left(\tau_{\mathrm{LMH}} \sim 7 \mathrm{~ns}\right)$ produites par mélange d'ondes dans une cellule de vapeur de mercure. Pour ce type de source, une difficulté majeure provient de la présence simultanée du signal VUV généré et des composantes du laser de pompage. Les détecteurs permettent la caractérisation de la forme et de l'intensité des impulsions VUV sans être perturbés par le signal simultané à $313 \mathrm{~nm}$. Ce type de détecteur a également été utilisé pour la métrologie d'impulsions $\mathrm{X}$ de basse énergie $(\mathrm{E}<1 \mathrm{keV})$ avec une résolution temporelle inférieure à $70 \mathrm{ps}$ en acquisition monocoup.
\end{abstract}

\section{INTRODUCTION}

Les sources de rayonnements UV et $\mathrm{X}$ ainsi que leurs applications sont actuellement en pleine expansion dans les domaines expérimentaux et industriels. Elles nécessitent le développement d'une instrumentation capable de réaliser la métrologie des rayonnements dans la gamme $10^{1}-10^{4} \mathrm{eV}$ peu couverte par les détecteurs commerciaux. Dans ce cadre, le LETI/DEIN du CEA Saclay est impliqué dans la mise au point de photodétecteurs en arséniure de gallium et en diamant couvrant un large domaine d'énergie compris entre $1 \mathrm{eV}$ et plusieurs dizaines de $\mathrm{MeV}$.

Des détecteurs ont été développés pour la métrologie dimpulsions subnanosecondes [1,2]. Ils sont réalisés à base d'arséniure de gallium (AsGa) et de diamant et permettent la mesure de l'intensité et de la forme des impulsions. Afin de réduire le temps de réponse des dispositifs en AsGa (de l'ordre de la nanoseconde) une étape de pré-irradiation du détecteur avec des neutrons de fission est réalisée. Elle conduit à l'augmentation de la concentration en défauts électriques dans le matériau et donc à la diminution de la durée de vie des porteurs et du temps de réponse des détecteurs. Les détecteurs ainsi obtenus ont des résolutions temporelles (largeur à mi-hauteur -LMH) inférieures à 70 ps en mode monocoup correspondant à la bande passante de l'oscilloscope $(7 \mathrm{GHz})$.

Cet article présente les résultats obtenus au Laboratoire de Physique des Lasers de l'Université de Villetaneuse avec des photodétecteurs "solar blind" spécifiquement réalisés pour la caractérisation d'impulsions VUV à $125 \mathrm{~nm}$ produites par mélange d'ondes dans une cellule de vapeur de mercure [3]. Une difficulté majeure dans la caractérisation de radiations VUV produites par génération d'harmoniques ou par mélange à quatre ondes provient de la présence simultanée du signal VUV et des composantes du laser pompe. Pour résoudre ce problème, nous avons développé des détecteurs à base de diamants peu sensible dans le domaine visible et UV $(\lambda>225 \mathrm{~nm})$. Les détecteurs permettent la caractérisation de la forme et de l'intensité des impulsions VUV à $125 \mathrm{~nm}$ sans être gênés par la présence simultanée des composantes du laser de pompage à 313 et $632 \mathrm{~nm}$. Le signal électrique obtenu à $125 \mathrm{~nm}$ est 500 fois supérieur à celui obtenu à $313 \mathrm{~nm}$, conduisant à une excellente sélectivité. Par ailleurs, ces détecteurs utilisés en mode monocoup offrent une résolution temporelle inférieure à 70 ps.

\section{PRINCIPE DE DETECTION}

Des photodétecteurs "solar blind", c'est à dire transparent dans le domaine visible et au delà dans l'UV ont été réalisés à partir de diamant non-dopé de haute résistivité. Le diamant présente un ensemble de propriétés exceptionnelles. Sa grande largeur de bande interdite $(5.5 \mathrm{eV}$, soit $225 \mathrm{~nm})$ le rend quasi transparent dans les domaines de l'infra-rouge, du visible et de l'UV jusqu'à $225 \mathrm{~nm}$. C'est cette propriété 
qui est à l'origine de l'effet "solar blind". La grande largeur de bande interdite permet également d'envisager la conception de détecteurs fonctionnant à haute température. Les fortes liaisons atomiques du carbone sous forme diamant lui assure une très bonne inertie chimique et une forte tenue aux rayonnements. La faible durée de vie des porteurs et leur mobilité élevée permettent la réalisation de dispositifs rapides et présentant une bonne sensibilité. Enfin, la faible absorption dans le domaine des $\mathrm{X}$ de basse énergie $\left(10^{1}\right.$ $10^{4} \mathrm{eV}$ ) conduit à une interaction rayonnement-matière plus en volume qu'avec les semi-conducteurs courants ( $\mathrm{Si}, \mathrm{GaAs}$ ) qui permet la réalisation de dispositifs pour la mesure en ligne des faisceaux et qui augmente le seuil de saturation en énergie des détecteurs.

Le dispositif de détection est constitué d'un échantillon de diamant sur lequel sont déposés deux contacts électriques qui permettent de polariser le détecteur (voir figure 1(a)). Lorsqu'une impulsion radiative traverse le détecteur, elle génère des paires électron-trous qui en dérivant sous l'effet du champ appliqué produisent une impulsion de courant aux bornes du détecteur. L'impulsion électrique délivrée aux bornes d'un oscilloscope $V_{\text {osc, }}$, est de la forme :

$$
V_{\text {osc }}=R q N_{\text {gen }} E\left(\mu_{\mathrm{n}} \tau_{\mathrm{n}}+\mu_{\mathrm{p}} \tau_{\mathrm{p}}\right) / l
$$

où $R$ est la résistance d'entrée de l'oscilloscope, $q$ la charge élémentaire, $N_{\text {gen }}$ le nombre de charge générées par seconde, $\mathrm{E}$ le champ électrique appliqué, $\mu_{\mathrm{n}}$ et $\mu_{\mathrm{p}}$ la mobilité des électrons et des trous, respectivement, $\tau_{\mathrm{n}}$ et $\tau_{\mathrm{p}}$ leurs durées de vies et $l$ la distance entre les électrodes. $\mathrm{N}_{\text {gen }}$ et $\tau$ sont contrôlés par les processus de génération et de recombinaison des porteurs. Ces derniers dépendent de la qualité cristallographique ainsi que des propriétés électroniques et optiques du matériau.

Pour des photons d'énergie supérieure à la bande interdite $(5.5 \mathrm{eV})$, les porteurs libres résultent de l'excitation intrinsèque d'électrons de la bande de valence à la bande de conduction. Pour les photons d'énergie inférieure à la bande interdite, les porteurs libres sont générés par excitation extrinsèque : génération d'électrons dans la bande de conduction à partir de niveaux profonds situés dans la bande interdite par exemple. Ces niveaux profonds sont dus à des impuretés ou défauts cristallographiques dans le diamant. L'azote par exemple est une impureté commune dans le diamant qui produit des niveaux situés à 1.7 et $3.83 \mathrm{eV}$ de la bande de conduction.

Pour réaliser des détecteurs "solar blind" en diamant, la quantité de porteurs générés par processus extrinsèque doit être négligeable devant la quantité de porteurs généré par processus intrinsèque sous irradiation. Ceci permet d'obtenir une bonne sélectivité entre le signal utile (signal VUV) et le signal simultané et parasite (signal UV). Pour atteindre cet objectif, les conditions de synthèse des films de diamant ont fait l'objet d'une étude approfondie afin d'optimiser la qualité du matériau.

\section{DISPOSITIF EXPERIMENTAL}

Les films de diamant polycristallin utilisés pour la fabrication des dispositifs de détection ont été synthétisés au DEIN par dépôt chimique en phase vapeur (CVD) assisté par plasma micro-onde $(2.45 \mathrm{GHz})$. Les dépôts sont réalisés sur substrats de $5 \mathrm{~cm}$ de diamètre, à haute température $\left(700-1000^{\circ} \mathrm{C}\right)$ et à partir d'un mélange de méthane $(0.5$ à $5 \%$ ) et d'hydrogène. Les paramètres de dépôt ont été optimisés afin d'obtenir des films transparents dans le domaine UV au dessus de $225 \mathrm{~nm}$ et présentant de bonnes propriétés électroniques (mobilité et durée de vie des porteurs) [4]. Les photoconducteurs ont été réalisés à partir de films de diamant de 20 à $150 \mu \mathrm{m}$ d'épaisseur. Des contacts électriques coplanaires ont été formés par évaporation d'or sur la surface du diamant pour réaliser des photoconducteurs ayant des surfaces actives comprises entre quelques $\mathrm{mm}^{2}$ et $1 \mathrm{~cm}^{2}$. Un détecteur en diamant naturel de type Ira, de haute pureté et présentant une très faible absorption dans le domaine UV-visible $(\lambda>225 \mathrm{~nm})$, a également été utilisé pour fabriquer un détecteur "solar blind" de référence. La figure 1(b) montre les photographies de la surface d'un photodétecteur en diamant à contacts interdigités et de son boîtier associé.

Les détecteurs ont été caractérisés sous impulsions VUV à $125 \mathrm{~nm}$ avec une source cohérente développée au Laboratoire de Physique des Lasers et dont la description est donnée en référence [5]. Le 

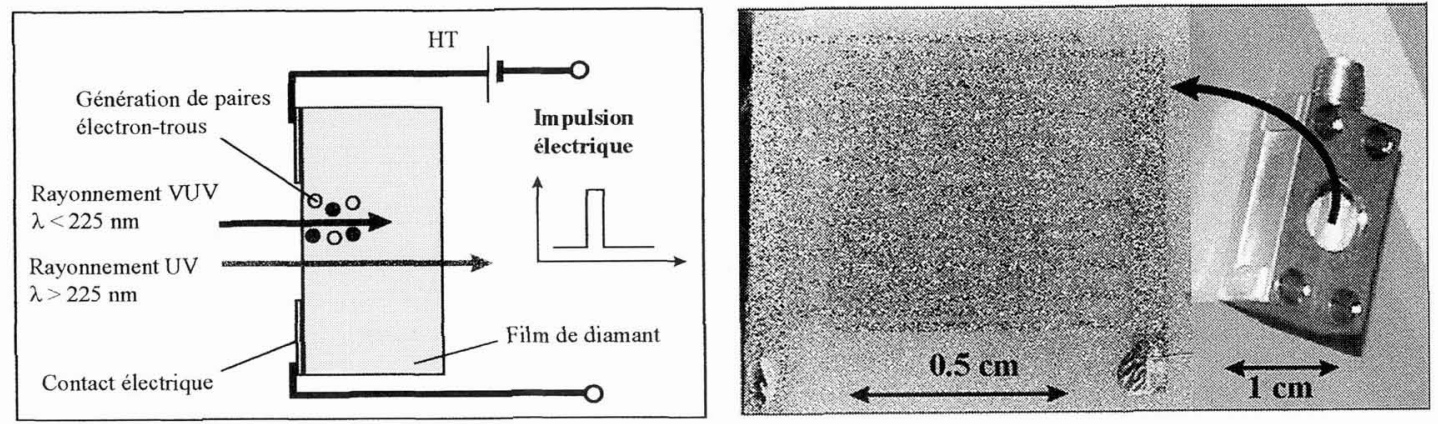

Figure 1: (a) Principe de détection des photoconducteurs "solar blind"; (b) Photographies de la surface d'un photodétecteur en diamant à contacts interdigités et de son boîtier associé.

système, stable et simple d'utilisation, est basée sur des effets non linéaires de mélange de fréquences à quatre ondes dans de la vapeur de mercure. La source fournie des impulsions VUV à $125 \mathrm{~nm}$ de $7 \mathrm{~ns}$ de largeur à mi-hauteur $(20 \mathrm{~Hz})$. En pratique, lorsque le détecteur est irradié, il reçoit simultanément le faisceau VUV ainsi qu'une part résiduelle des faisceaux de pompage sous la forme de lumière diffusée à 313 et $625 \mathrm{~nm}$.

Les diamants ont été testés sous différentes conditions pour évaluer la réponse dans le visible, l'UV

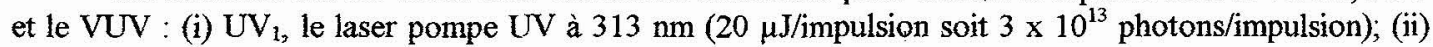
VUV+ $\mathrm{UV}_{2}$, le faisceau complet en sortie de cuve à mercure $(\lambda=125 \mathrm{~nm}$ avec une énergie de $40 \mathrm{~nJ} /$ impulsion, soit $2.4 \times 10^{10}$ photons/impulsion, en présence de lumière diffusée, $\lambda=313 \mathrm{~nm}$ avec $1 \mu \mathrm{J} / \mathrm{impulsion}$, soit $1.7 \times 10^{12}$ photons/impulsion) et (iii) $\mathrm{UV}_{2}$, la lumière diffussée seule $(\lambda=313 \mathrm{~nm}$ et $1 \mu \mathrm{J} /$ impulsion). Les mesures ont été réalisée avec un oscilloscope $1 \mathrm{GHz}$. Des mesures complémentaires sous irradiation $X$ pulsée de basse énergie $\left(E \leq 1 \mathrm{keV}, \tau_{\mathrm{LHM}}=30 \mathrm{ps}\right)$ ont été également réalisées afin d'évaluer le temps de réponse des détecteurs. Pour ces mesures, les détecteurs étaient connectés à un oscilloscope ultra-rapide Intertechnique 7000 , avec une bande passante de $7 \mathrm{GHz}$ (temps de montée $\sim 30 \mathrm{ps}$ ).

\section{RESULTATS ET DISCUSSION}

La figure 2(a) présente les signaux transitoires obtenus sous irradiation simultanée à $125 \mathrm{~nm}$ et $313 \mathrm{~nm}$ $\left(V U V+U V_{1}\right)$ avec deux photoconducteurs en diamant CVD et en diamant naturel pour comparaison. Les détecteurs ont été polarisés sous $200 \mathrm{~V}$. Ils permettent d'obtenir des impulsions avec une tension crête de $2.8 \mathrm{mV}$ et $1.7 \mathrm{mV}$, respectivement. Par ailleurs, les deux détecteurs ne fournissent pas de signal significatif sous faisceau UV diffusé seul à $313 \mathrm{~nm}\left(\mathrm{UV}_{1}\right)$. Le signal obtenu sur la figure 1 résulte donc principalement de la radiation VUV à $125 \mathrm{~nm}$, bien que celle émise à $313 \mathrm{~nm}\left(U V_{1}\right)$ soit 25 fois plus intense. L'effet "solar blind", jusqu'à des longueurs d'onde de l'ordre de $225 \mathrm{~nm}$, dans le diamant permet donc de caractériser le faisceau VUV sans être perturbé par l'émission simultanée des composantes de pompage. Ce haut pouvoir de sélectivité entre rayonnements UV et VUV résulte de la bonne qualité du diamant naturel de type IIa et du diamant CVD synthétisé avec des procédés optimisés. Les propriétés optiques (faible absorption) et électriques (faible concentration de défauts profonds) contribuent à la génération d'une faible concentration de porteurs par excitation extrinsèque et donc à un signal négligeable provenant de l'émission à $313 \mathrm{~nm}$.

Une mesure précise de la sélectivité des photodétecteurs a été réalisée en irradiant les dispositifs avec le faisceau direct à $313 \mathrm{~nm}\left(\mathrm{UV}_{2}\right)$. Les valeurs de sensibilité mesurée à 125 et $313 \mathrm{~nm}$ sont respectivement de 9500 et $21 \mathrm{nC} / J$ pour le détecteur en diamant IIa et 13000 et $35 \mathrm{nC} / \mathrm{J}$ pour un détecteur en diamant CVD. La sensibilité à $125 \mathrm{~nm}$ est donc environ 500 fois plus élevée à $125 \mathrm{~nm}$ qu'à $313 \mathrm{~nm}$, procurant une excellente sélectivité pour la caractérisation du faisceau VUV. La sélectivité légèrement moins élevée des détecteurs en diamant CVD ( 360 ), par rapport à celle du diamant naturel (x 450), indique que le 

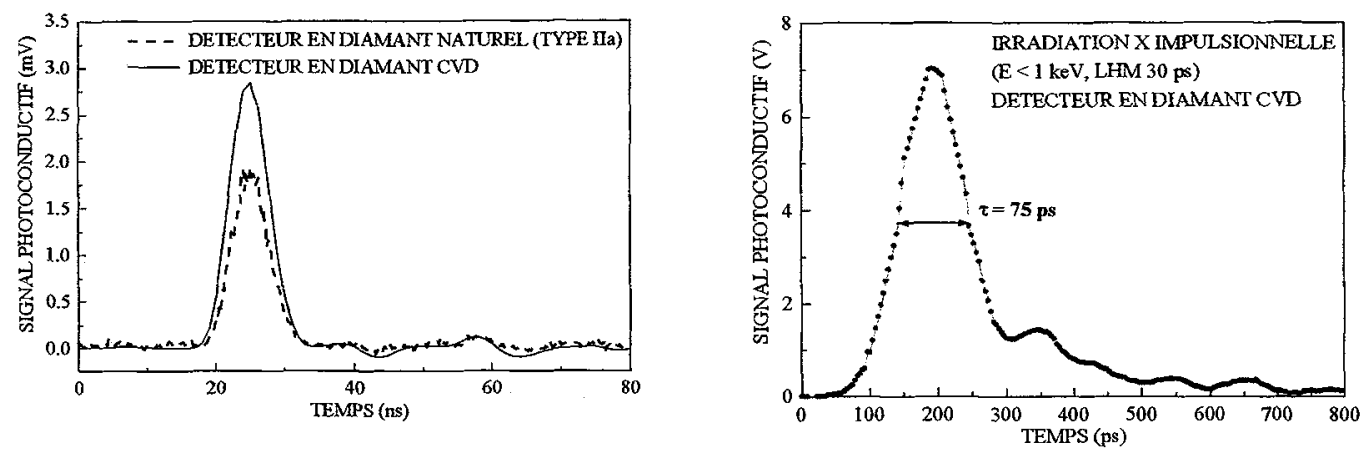

Figure 2 : (a) Signaux transitoires obtenus sous irradiation simultanée à $125 \mathrm{~nm}$ et $313 \mathrm{~nm}\left(\mathrm{VUV}_{+} \mathrm{UV} \mathrm{V}_{\mathrm{l}}\right.$ avec un photoconducteurs en diamant $\mathrm{CVD}$ et un en diamant naturel $\left(\mathrm{V}_{\mathrm{pol}}=200 \mathrm{~V}\right)$. Les détecteurs "solar blind" ne présentent aucun signal mesurable sous irradiation à $313 \mathrm{~nm}$ seule $\left(\mathrm{UV}_{1}\right)$; (b) Signaux transitoires obtenus sous irradiation $\mathrm{X}$ impulsionnelle $\left(\mathrm{E}<1 \mathrm{keV}, \tau_{\mathrm{LHM}}=30 \mathrm{ps}\right)$ avec un détecteur en diamant $\mathrm{CVD}$.

matériau synthétique possède une concentration en défauts profonds plus élevée qui favorise la génération de porteurs par excitation extrinsèque. Il faut cependant noter que les propriétés électriques des films de diamant CVD sont comparables à celles des diamants naturels de type IIa de qualité standard. Une étude est en cours afin d'améliorer encore la sélectivité des films de diamant en réduisant l'incorporation d'impuretés durant le dépôt et donc la densité de niveaux de pièges dans la bande interdite du diamant.

La figure 2(b) montre le signal photoconductif obtenu avec un détecteur en diamant CVD polarisé à 100 volts sous impulsion $X$ de basse énergie $(E<1 \mathrm{keV})$. Il présente une largeur à mi-hauteur de $70 \mathrm{ps,}$ correspondant à la résolution temporelle de l'oscilloscope utilisé et montre la possibilité d'utiliser les détecteurs en diamant CVD pour la caractérisation d'impulsions $X$. L'utilisation de technique d'échantillonage avec des détecteurs en diamant CVD doit par ailleurs permettre d'accéder à des temps de réponse inférieurs à la dizaine de picosecondes.

\section{CONCLUSION}

Des photodétecteurs "solar blind" en diamant CVD ont spécifiquement été développés pour la caractérisation d'impulsions VUV à $125 \mathrm{~nm}$ produites par mélange d'ondes dans une cellule de mercure. Ils offrent une excellente sélectivité entre le signal utile et les composantes du laser de pompage à 313 et 632 $\mathrm{nm}$. Par ailleurs, ces détecteurs utilisés en mode monocoup offrent une résolution temporelle inférieure à $70 \mathrm{ps}$ (bande passante de $7 \mathrm{GHz}$ de l'oscilloscope utilisé). Les études menées au LETI/DEIN sur la synthèse de films de diamant par dépôt chimique en phase vapeur (CVD) ouvrent ainsi de nouvelles possibilités dans la réalisation de photodétecteurs en diamant pour la métrologie d'impulsions et la mesure de flux dans le domaine UV-X $\left(10^{1}-10^{4} \mathrm{eV}\right)$.

\section{Références}

1. F. Foulon, B. Brulot, C. Rubbelynck, P. Bergonzo, T. Pochet; IEEE Trans. Nucl. Sc. 43, 1372 (1996).

2. F. Foulon, P. Bergonzo, C. Jany, A. Gicquel, T. Pochet; Nucl. Instr.and Meth. A380, 42 (1996).

3. F. Foulon, P. Bergonzo, C. Borel, R.D. Marshal, C. Jany, L. Besombes, A. Brambilla, D. Riedel, L. Museur, M.C. Castex, A. Gicquel, J.Appl. Phys. 84, 5331 (1998).

4. C. Jany , F. Foulon, P. Bergonzo, B. Guizard, C. Borel, A. Brambilla, S. Haan, A. Tardieu, A. Gicquel, Electrochemical Society Proceeding, Vol 97-32, 208(1998).

5. L. Museur, W.Q. Zheng, A. Kanaev and M.C. Castex, IEEE J.of Sel.Topics in QEI. 1, 900 (1995). 